Revisão de literatura - 361 -

\title{
TRABALHO, EDUCAÇÃO E POLÍTICA EM SEUS NEXOS NA PRODUÇÃO BIBLIOGRÁFICA SOBRE O CUIDADO ${ }^{1}$
}

\author{
Flávia Regina Souza Ramos², Judite Hennemann Bertoncini³, Rosani Ramos Machado ${ }^{4}$, Rita de Cássia Flor 5 , \\ Denise Elvira Pires de Pires ${ }^{6}$, Francine Lima Gelbcke ${ }^{7}$
}

${ }^{1}$ Trabalho realizado com apoio do CNPq / CAPES.

${ }^{2}$ Doutora em Enfermagem. Professor Associado do Departamento de Enfermagem e do Programa de Pós-Graduação em Enfermagem (PEN) da Universidade Federal de Santa Catarina (UFSC). Pesquisadora CNPq. Santa Catarina, Brasil. E-mail: flavia.ramos@pq.cnpq.br

${ }^{3}$ Doutoranda do PEN/UFSC. Professora do Departamento de Enfermagem da Fundação Universidade Regional de Blumenau. Santa Catarina, Brasil. E-mail: juditeb@furb.br

${ }^{4}$ Doutoranda do PEN/UFSC. Docente da Universidade do Vale do Itajaí Enfermeira da Secretaria de Estado da Saúde de Santa Catarina. Santa Catarina, Brasil. E-mail: rosaniramos@terra.com.br

${ }^{5}$ Doutoranda do PEN/UFSC. Docente do Centro Federal de Educação Tecnológica de Santa Catarina. Santa Catarina, Brasil. E-mail: flor@newsite.com.br

${ }^{6}$ Doutora em Ciências Sociais. Professor Associado do Departamento de Enfermagem e do PEN/UFSC. Santa Catarina, Brasil. E-mail: piresdp@yahoo.com

${ }^{7}$ Doutora em Enfermagem. Professor Associado do Departamento de Enfermagem e do PEN/UFSC. Santa Catarina, Brasil. E-mail: fgelbcke@ccs.ufsc.br

\begin{abstract}
RESUMO: Trata-se de revisão bibliográfica que analisou 57 artigos acerca do cuidado, localizados a partir da base de dados LILACS. Teve como objetivo reconhecer as bases teóricas e tendências desta produção. Este artigo apresenta os resultados relativos a categoria empírica "concepção histórico social do cuidado em sua relação com o trabalho, educação e política", pela relevância com que emergiu no conjunto da produção sobre o cuidado. As categorias trabalho, educação e política mostraram importantes relacionamentos nas posições críticas ou propositivas de alternativas teóricas para o cuidado em saúde e de enfermagem. Em síntese, a compreensão crítica sobre o trabalho aparece justificada por sua complexidade e por ser condição mobilizadora de interessses profissionais e sociais em torno do cuidado. O trabalho se mostra como categoria imprescindível para a instrumentalização teórico-política da ação profissional concretizada no cuidado, que por sua vez, pela via da apropriação crítica do trabalho, pode ser portador de novos valores e configurações.
\end{abstract}

DESCRITORES: Trabalho. Enfermagem. Filosofia em enfermagem. Cuidados de enfermagem.

\section{WORK, EDUCATION, AND POLITICS IN ITS LINKS TO BIBLIOGRAPHIC PRODUCTION CONCERNING CARE}

\begin{abstract}
This is a bibliographic review which analyzed 57 publications concerning care located in the LILACS database. The objective of this review was to recognize the theoretical bases and trends of this production. The article presents results related to the "social-historical conception" empirical category in its relation to work, education, and politics through its relevance to what emerged in a set of scientific production concerning care. The categories work, education, and politics showed important relationships in critical positions or proposals for theoretical alternatives for health and nursing care. Critical comprehension about work appears to be justified by its complexity and for being a mobilizing condition of professional and social interests about care. Work has shown itself to be an essential category for theoretical-political instrumentalization of professional action, made real through care, which in turn, through critical appropriation of work, may be a carrier for new values and configurations.
\end{abstract}

DESCRIPTORS: Work. Nursing. Philosophy, nursing. Nursing care.

\section{TRABAJO, EDUCACIÓN Y POLÍTICA EN SUS NEXOS CON LA PRODUCCIÓN CIENTÍFICA SOBRE EL CUIDADO}

RESUMEN: Se trata de una revisión bibliográfica, en la cual se analizaron 57 artículos acerca del cuidado, localizados en la base de datos LILACS. El objetivo fue reconocer las bases teóricas y las tendencias de esa producción científica. En este artículo se presentan los resultados relativos a la categoría empírica: concepción histórico-social del cuidado en su relación con el trabajo, la educación y la política, por la relevancia con la cual esa categoría surgió en el conjunto de la producción sobre el cuidado. Las categorías trabajo, educación y política mostraron importantes relaciones en las posiciones críticas o proponentes de alternativas teóricas para el cuidado en salud y en enfermería. En síntesis, se puede decir que la comprensión crítica sobre el trabajo aparece justificada por su complejidad y por ser condición para movilizar intereses profesionales y sociales alrededor del cuidado. El trabajo se muestra una categoría imprescindible para la instrumentalización teórico-política de la acción profesional manifestada en el cuidado que, a su vez, por la vía de la apropiación crítica del trabajo, puede ser portador de nuevos valores y configuraciones.

DESCRIPTORES: Trabajo. Enfermería. Filosofía en enfermería. Cuidados de enfermería. 


\section{INTRODUÇÃO}

De muitos lugares e posições os sujeitos expressam visões sobre o objeto de seus trabalhos, e demarcam territórios teóricos e profissionais que, em última análise, manifestam alguns interesses litigantes mas, também as interfaces e movimentos de saberes e práticas em fronteiras móveis e em permanente negociação.

A produção bibliográfica sobre o cuidado é reveladora de conflitos e dissensos em torno de modelos de assistência, projetos profissionais, saberes e poderes nas relações entre as profissões e os campos de saber e prática.

A vitalidade desse cenário científico e o modo como nele se processam movimentos intelectuais e propostas práticas para o trabalho em saúde, motivou o estudo "A produção científica sobre o cuidado no campo da saúde - bases teóricas e tendências". O primeiro suposto foi o de considerar o cuidado como uma ação e um produto em permanente reconstrução, de grande importância ao se pensar o trabalho em saúde. Um segundo suposto foi reconhecer a problematização deste discurso sobre o cuidado como empreendimento intelectual fundamental, que deve incorporar desdobramentos éticos, políticos e tecnológicos. E, ainda, que a produção acadêmico-científica pode ser entendida como documentos constituintes de discursos eficazes, já que conferem aos sujeitos que o proferem uma perspectiva de lugar, competência e legitimidade.

O estudo que originou o presente artigo buscou responder a alguns dos desafios emergentes na produção do conhecimento sobre o trabalho em saúde e em enfermagem, objetivando analisar a produção bibliográfica sobre o cuidado no campo do trabalho multiprofissional em saúde, especialmente, reconhecendo as bases teóricas e tendências desta produção.

A intersecção entre as categorias trabalho, política e educação com a produção bibliográfica sobre o cuidado foi uma das categorias que se destacou nesta pesquisa, por isso mereceu o aprofundamento analítico que resultou neste texto. Essa intersecção e a relevância com que estes temas aparecem no âmbito teórico e das práticas profissionais, justifica o estudo. Portanto, este artigo tem como objetivo estabelecer os nexos entre as dimensões do trabalho, da educação e da política com o cuidado, uma vez que este cuidado mantém uma interlocução com o mundo do trabalho. Interlocução ora investida da dimensão política, anunciada pelas escolhas de quem faz ou deveria fazer o cuidado, sobre para quem é realizado o cuidado e para qual finalidade se destina e, ora investida da dimensão educativa expressa na mesma relação com o trabalho.

\section{O DESENHO METODOLÓGICO}

Trata-se de uma revisão bibliográfica que buscou uma análise ampla e sistemática de estudos sobre o tema, permitindo sua caracterização e a apreciação das bases teóricas e tendências da produção relacionada ao cuidado.

O levantamento bibliográfico foi realizado através de consulta à BIREME, na base de dados do LILACS. A busca na base de dados foi realizada durante os meses de março e abril de 2007, considerando como descritores de busca: assistência à saúde, cuidados médicos, assistência integral à saúde e cuidado de enfermagem. O primeiro levantamento possibilitou a identificação de 22.159 registros, os quais foram refinados a partir do critério de convergência com o objetivo do estudo. Salienta-se que o principal critério de inclusão foi a identificação de uma proposição teórica para o cuidado, uma reflexão ou análise conceitual. Assim, justifica-se a exclusão da grande maioria dos resumos, por tratar-se de estudos sobre práticas de cuidado aplicado a áreas ou temas específicos, sem necessariamente promover uma análise do conceito de cuidado ou de sua abordagem no plano mais geral das práticas sociais. Dessa forma, chegou-se ao total de 68 registros (entre capítulos de livros e artigos). Destes, foram localizados os textos integrais de 57 estudos, que passaram a compor as fontes documentais do processo de análise.

Como instrumento de coleta de dados foi elaborado uma ficha de análise a qual permitiu ordenar os principais achados em torno de quatro categorias principais: a) concepção de cuidado; b) referencial teórico-metodológico; c) contribuição para a construção de um pensamento sobre o cuidado; d) especificidade da contribuição para as práticas no campo da saúde. Para cada uma dessas categorias o analista do artigo descrevia sua interpretação e incluía trechos do artigo representativos desta compreensão. Do conjunto dos 57 instrumentos preenchidos, todos os achados referentes a cada categoria foram reunidos e sofreram releitura e discussão pelo conjunto de pesquisadores que participaram da primeira análise. Neste momento os dados passaram por uma segunda categorização, que produziu conjuntos menores de artigos ou de corpus documentais, agrupados por tal pertinência com uma forma de 
contribuição ou reflexão. Este conjunto menor de artigos foi novamente lido na íntegra, de modo a apreender a especificidade desta contribuição.

Assim, elegeu-se explorar neste artigo o cuidado em sua relação com a educação, o trabalho e a política, sendo esta uma das contribuições identificadas nos estudos. O corpus documental do eixo ora tematizado constituiu-se de 15 artigos na forma de reflexão e/ou ensaio teórico, sendo que destes, 14 foram escritos por enfermeiras(os) e um em parceria entre enfermeira e outros profissionais. Quanto ao país de origem, todos os textos foram produzidos por autora(es) brasileiras(os), vinculadas a instituições públicas (90\%).

\section{RELAÇÃO TRABALHO, EDUCAÇÃO E POLÍTICA COMO CONDIÇÃO DA PRÁ- XIS DO CUIDADO}

No conjunto das produções analisadas fica evidente a contribuição de um conjunto de estudos que emergiram nos anos 80 e tiveram forte impacto na enfermagem brasileira, alinhados à vertente teórica do materialismo histórico. Esta produção mobilizou a análise do processo de trabalho em saúde como prática social sob o ângulo da crítica ao modo de produção capitalista e de suas conseqüências sobre o modo de trabalhar e organizar o setor saúde, como os estudos realizados. ${ }^{1-4}$

O que fica claro neste conjunto de estudos é a superação de uma concepção essencializada (natural, fundamental e indispensável, em si mesma, imutável) para a consideração do caráter histórico e cultural das práticas sociais nas quais o cuidado se desenvolve e é, inclusive, objeto de construção do pensamento. No interior deste conjunto muito amplo de estudos, ainda são diferenciadas as formas de abordar o cuidado de acordo com o modo como se analisa a própria ação em foco, como prática social ou processo de trabalho, como arte ou tecnologia de cuidar, por exemplo.

A análise dos artigos remeteu a conexão das categorias educação, trabalho e política como condição da práxis do cuidado. Esta ligação se expressa em diferentes perspectivas como:

- na defesa da articulação das dimensões técnica, política, comunicativa e de desenvolvimento da cidadania. Alguns artigos apontam que a política e a ciência podem construir valores que permitam coerência entre a subjetividade e a realidade, para resgatar a cidadania pela consciência de si e do outro. Em nossa análise, em argumentos como este há que se manter certas precauções contra a idéia positivista de uma realidade determinada por leis objetivas e neutras, em favor de uma concepção de subjetividade que se expressa objetivamente, como estruturante e constitutiva da realidade. Assim, discursos de crítica às visões tradicionais podem, ainda, reforçar antigas cisões, como de uma subjetividade essencial e alheia aos processos sociais, mesmo que na defesa de uma suposta meta política de engajamento e transformação;

- nas tentativas de desconstrução do modelo da biomedicina e na construção de cuidado coletivo com base no modelo epidemiológico. ${ }^{5} \mathrm{O}$ modelo epidemiológico é apontado como potente para expandir o modelo clínico tradicionalmente usado para orientar as práticas assistenciais sustentadas pelo esquartejamento anatômico e fisiológico do corpo. Mesmo que reconhecido em sua potencialidade, como acontece em produções representativas, parecia ainda inicial a crítica sobre seus próprios limites. $\mathrm{O}$ modelo epidemiológico, ainda que fundamental, quando usado de forma isolada também possui limites para explicação e solução dos problemas a serem enfrentados na produção do cuidado; mas a contraposição ao modelo clínico e seus reflexos na ordenação das práticas foi o alvo "exclusivo", que assegurou certa afinidade com modelos que, só mais tarde, também sofreriam uma apropriação mais crítica. Neste sentido, concordamos que a transgressão das fronteiras dos cuidados médicos tradicionais é fator decisivo na ampliação do território do cuidado. Assim, instituir outras formas de tratar e cuidar que não sejam exclusivas da biomedicina apresenta potencial emancipador e transformador das tradicionais relações sociais existentes no cotidiano dos serviços de saúde; ${ }^{6}$

- no trabalho interdisciplinar, sugerido como alternativa para superar o modelo clínico biológico e resgate da clínica ressignificada pela voz dos usuários, como o cuidado que vem sendo denominado de clínica ampliada ou clínica do sujeito, por profissionais de saúde, notadamente médicos; ${ }^{7-8}$

- na crítica ao cuidado de enfermagem quando limitado a uma ação tecnicamente competente, relacionando-o, apenas, a disciplinar indivíduos e complementar o ato médico. Isto fundamentaria práticas sobre a doença e partes do corpo doente, modelo construído com base em disciplinas como anatomia e fisiologia, bastante problematizado na maioria dos estudos;

- na proposição do cuidado integral e no que este implica em termos de organização dos trabalhadores de enfermagem, programas de edu- 
cação continuada para os seus agentes, parcerias entre instituições de ensino, serviços e entidades de classe para formular e implementar política de recursos humanos em enfermagem. ${ }^{5}$ A dimensão política se evidencia pelo foco nas características e condições do exercício profissional da enfermagem e em situações a serem enfrentadas no âmbito das políticas públicas, para viabilizar um cuidado de qualidade à população. Corroboramos a pertinência deste enfoque, já que só a representação quantitativa da enfermagem no conjunto dos trabalhadores no setor saúde público e privado, justificaria tal preocupação por parte dos gestores;

- na articulação dos conceitos de cuidado e trabalho. O cuidado de enfermagem aparece como fato social e objeto de trabalho dos enfermeiros. Aqui, alguns autores fazem um contraponto elucidativo ao tratarem o cuidado como objeto epistemológico e não objeto de trabalho da enfermagem, pois o cuidado é o próprio ato em resposta a alguma necessidade de atenção; ${ }^{9}$

- no privilegiamento da dimensão micropolítica, quando se propõe a construção de um espaço de encontro nos serviços de saúde, possibilitando o surgimento de novos saberes e práticas de saúde que respondam à demanda existente por cuidado. Isso depende, contudo, da porosidade desses espaços aos modos de andar a vida dos sujeitos neles implicados de forma que se possa constituir como cenários de emancipação e de construção de movimentos contra-hegemônicos;

- no trabalho individual ou coletivo enquanto ação independente, aquela que permite tomada de decisão somente da enfermeira, e/ou ações interdependentes, as que são complementares aos atos de outros profissionais e que podem ter diferentes dimensões, dada às relações de poder e à divisão do trabalho na equipe de saúde. ${ }^{10}$

- no compromisso com uma filosofia ainda por ser construída, na qual o cuidado possa se expor ao diálogo no âmbito de toda a sociedade, no esforço por tornar essas questões estratégicas para a sobrevivência humana e do planeta.

\section{TRABALHO COMO CATEGORIA FUN- DAMENTAL NA CONSTRUÇÃO DO CUIDADO}

A compreensão crítica sobre o trabalho se justifica por sua complexidade e por ser condição mobilizadora de interesses profissionais e sociais em torno do cuidado. O trabalho se coloca como categoria imprescindível para a instrumen- talização teórico-política da ação profissional, concretizada no cuidado; este cuidado, por sua vez, poderia ser portador de valores novos e diferenciados ao articular-se, criticamente, com a categoria trabalho.

As justificativas para a proposição desta compreensão do trabalho em sua complexidade, pode ser exemplificada por um discurso que remete às mudanças no mundo do trabalho - como aumento da especialização, competição com outras profissões congêneres, habilidade com novas tecnologias - para mostrar a necessidade de novo olhar sobre a prática da enfermagem, trazendo grandes desafios para a profissão. ${ }^{11} \mathrm{Ou}$, ainda, quando a importância da articulação das dimensões técnica, política, comunicativa e de desenvolvimento de cidadania no trabalho de cuidar e de gerenciar o cuidado é tomada na tematização da tecnologia no cuidado/trabalho. Destaca-e aí o elemento político das escolhas tecnológicas e a demanda por recompor a utilização de tecnologias duras, leve-duras e leves. ${ }^{12}$

O cuidado como meta e o trabalho como categoria estratégica se justifica, também, quando se argumenta que o processo de trabalho em saúde deveria orientar a análise e práticas profissionais de cuidado, a partir de perguntas como: quem faz o cuidado? Como se dá a divisão do trabalho para a efetivação do cuidado? A quem o cuidado se destina? A que finalidade o trabalho de cuidar se submete? Sob que condições os trabalhadores desenvolvem o cuidado? ${ }^{5}$

Os exemplos acima descritos justificam a relevância da perspectiva teórica que relaciona trabalho e cuidado assim como a pauta políticocientífica que diz respeito aos fins e metas do cuidado, destacando os sujeitos destas metas e pautas. Aqui se mostram, ainda, sinais do que poderia ser pensado como inconsistências e incoerências dos discursos, mas que também podem se pensados como sinais de confrontos históricos da profissão no esforço por legitimar-se no campo do conhecimento científico e das próprias práticas sociais. Também há que se considerar que se no conjunto o discurso pode revelar tais contradições, a idéia de um todo uniforme e coeso só muito abstratamente pode ser exercitada. Em alguns aspectos pode-se configurar certos pontos de ligação e adesão, no caminho de um discurso comum e compartilhado, mas um conjunto bem maior de diferenças, desarticulações e bifurcações se abrem das vias largas deste senso unificador. Neste sentido, não se poderia falar de um discurso, 
mas de diferentes discursos tensionando posições e pensamentos, abrindo novas vias e elegendo focos privilegiados de análise. Assim, por exemplo, se o trabalho interdisciplinar foi manifestado como uma das formas reveladoras do nexo das categorias educação, trabalho e política nas proposições sobre o cuidado, isto não significa que não seja a interdisciplinaridade colocada em xeque por um conjunto de outros supostos, mesmo que nunca explicitamente contrários a ela.

Isto se mostra, exemplarmente, quando os autores, apesar de não reivindicarem o cuidado como exclusivo da Enfermagem, também em nenhum momento incluem outras profissões como praticantes do cuidado em saúde. Configura-se, assim, um discurso que "convoca" os profissionais da Enfermagem para produzir mudanças no perfil do cuidado, caracterizando-o como um compromisso autêntico na mudança social e estrutural da sociedade. Esse discurso alega a premência da tarefa e conclui sobre um outro compromisso, agora com a defesa da profissão sob outras bases, alegando que não se pode esperar mais, pois, se nada for feito para mudar esse panorama, "com certeza, outros profissionais irão ocupar este espaço em branco". ${ }^{13: 28}$

Finalmente, no que se refere à categoria trabalho, há referências à saúde do trabalhador como objeto de preocupação, numa concepção de que cuidado implica em preocupação e zelo com o ser humano trabalhador e na elaboração de políticas de cuidado para promoção da saúde do trabalhador. ${ }^{14}$

\section{POLÍTICA COMO ELEMENTO DA RELA- ÇÃO TRABALHO E CUIDADO}

O elemento político emerge nas discussões do cuidado como produto do trabalho em contextos complexos e conflitantes; como serviço ou bem social que impõe tarefas políticas em termos de direito e justiça; como espaço de poder no terreno das relações entre sujeitos diferenciados. Embora os exemplos possam parecer escassos, entendemos que eles são expressões representativas de certas perspectivas e suas nuances presentes no campo da saúde e da enfermagem, como se evidencia nos exemplos a seguir.

Para alguns autores, as práticas de cuidado são fortemente influenciadas pelas condições objetivas de sua produção que, ao se materializarem, expressam forças e contradições do jogo de saber/ poder e da ciência/técnica, a que se submetem, ou resistem, no contexto da sociedade hierarquizada na qual vivemos. ${ }^{15}$

Outros estudos destacam a importância do cuidado não só no plano individual, mas também no plano social. Como bem social deve ser eqüitativamente distribuído, tarefa esta possível apenas quando coletivamente empreendida.,

Especialmente na Enfermagem está presente a preocupação em evidenciar os espaços das relações do profissional com o usuário/paciente na perspectiva dos direitos, da cidadania e da autonomia. Obviamente que isto seria mais ricamente tratado, se o foco a partir do qual a produção fosse levantada, tivesse sido da ética/bioética, mas mesmo se tratando da construção teórica sobre o cuidado, o tema do poder mostrou-se em sua relação com o trabalho e, especialmente, em um demanda política do agir profissional. Propõe-se, por exemplo, a discussão das práticas de cuidado que negam a autonomia e a condição de cidadão, indicando a necessidade de repensar as posturas profissionais autoritárias, detentoras do poder e de um agir transformador. ${ }^{17}$ Vale ressaltar que o poder, neste caso, é retratado como um atributo centralizado e exercido/possuído por uns em detrimento de outros, mas que reconhece uma estreita relação com o saber, ou seja, o saber profissional na base da autoridade e da legitimidade, como também no uso que a ele dá pelo autoritarismo e abuso.

Para a construção do cuidado, tão importante quanto investir na reflexão e transformação das interações interpessoais nos atos assistenciais, é debruçar-se, uma vez mais, sobre as raízes e significados sociais dos adoecimentos em sua condição de obstáculos coletivamente postos a projetos de felicidade humana, e de forma articulada, da disponibilidade social de tecnologias e serviços necessários para a sua superação. Nesse sentido, é relevante na produção sobre o cuidado, a articulação de iniciativas teóricas e práticas que vinculem os cuidados individuais a aproximações de corte sócio-sanitário (diagnóstico de situação, planejamento de ações e monitoramento de processos e resultados relativos a determinado agravo ou situação de grupos populacionais específicos, organização dos serviços e os arranjos tecnológicos para respostas sanitárias num sentido mais geral). ${ }^{18}$

O elemento político se vislumbra na necessária compreensão de novas formas de cuidado integral nos espaços públicos de saúde, a partir das perspectivas de transformação do modelo hegemônico que estrutura as instituições de saúde 
e o próprio reconhecimento da profissão. Daí se desdobram denúncias, mobilizações e bandeiras em torno: - do cuidado como compromisso social do profissional de enfermagem; - do cuidado de enfermagem em suas determinações e contexto social; - do conformismo dos profissionais da enfermagem com padrões impostos como um problema ético que afeta negativamente o exercício do cuidado; - da necessidade de verdadeira inserção do profissional de enfermagem na sociedade, por meio de ações que promovam a cidadania. ${ }^{13}$

\section{EDUCAÇÃO COMO DIMENSÃO INEREN- TE AO CUIDADO E AO TRABALHO}

A categoria educação aparece em muitos artigos analisados como uma via de duplo sentido. Educação é uma forma de cuidar e o cuidado é uma forma de educar. Uma educação que potencializa o cuidar deve estar assentada no diálogo e, desta forma pode constituir-se em efetivo cuidado. ${ }^{18}$ Também é apontada a importância da educação em saúde na prática/cuidado de enfermagem como mediadora de processos de aprendizagem, como produção positiva (resistência, não obediência) a fim de modificar a condição de sujeição dos clientes, tendo uma postura mais ética e comprometida com a vida. ${ }^{19}$

Ao mesmo tempo que sugerem o cuidado como processos educativos de mediação e experiência de doença, ainda há referências nas quais a educação aparece reduzida à transferência de informações do saber profissional para a pessoa e esta, por si, mudaria sua atitude diante da experiência em relação aos serviços de saúde. Assim, os profissionais continuam sabendo o que é certo, detentores da verdade, mesmo que o certo agora seja resistir. O profissional teria o papel de decodificar o saber latente do cliente. Corre-se o risco de querer decodificar o saber latente do cliente para nós, profissionais, reconhecê-lo somente quando passível de ser traduzido na linguagem técnica. É fácil cair na armadilha da circularidade do discurso (profissional) na tentativa de buscar sua legitimação. A resistência como produção positiva é mais uma tese, assim como a obediência como valor negativo, e pode escamotear a prepotência do profissional mandatário de uma outra verdade científica, em nome da autonomia do sujeito/cliente.

Nesta mesma direção, alguns estudos contribuem com a proposição de uma educação para o cuidado, ${ }^{18,20}$ capaz de questionar o saber hegemônico como verdade, aquela que prioriza o desenvolvimento humano, cuja experiência é valorizada e aquela na qual os seres se expressam livremente e exercitam cidadania. É uma educação na qual existe participação, envolvimento e respeito. Ela oferece substrato para o cultivo do espírito humano em sua plena consciência e ação ética frente ao mundo. A educação para o cuidado ou como cuidado está fortemente associada ao exercício da liberdade e cidadania. ${ }^{19}$

Os processos de formação dos profissionais da saúde também foram objeto de análise nesses estudos considerando pelo menos dois aspectos. O primeiro é a necessidade de incluir nos currículos disciplinas como antropologia da saúde para fundamentar o processo de cuidar, na perspectiva sócio-cultural. ${ }^{21} \mathrm{O}$ segundo aspecto critica a valorização excessiva nos currículos do uso das tecnologias materiais (equipamentos e aparelhos) em detrimento das tecnologias não-materiais nos processos de cuidar. Novamente está em evidência a inadequação dos pressupostos do modelo biomédico para a formação profissional na área da saúde. Desta forma, sugerem o enfrentamento do que chamam de paradoxo da educação, de modo a integrar e flexibilizar estes pólos contrários: de um lado a alta tecnologia (tecnologia material), a produtividade e a competitividade e, de outro, a consideração do processo interativo e não meramente técnico do cuidado, que implica na revisão de valores mais humanísticos, inclusive retorno e reconhecimento da espiritualidade. O uso majoritário de tecnologias materiais empobrece a ação cuidadora e limita o seu alcance, apontando o potencial das tecnologias não-materiais para ações de enfermagem mais qualificadas, no processo de trabalho de cuidar e gerenciar o cuidado. ${ }^{12}$

Importante ressaltar que muitas referências à dimensão educativa não deixam de tratar do trabalho, o que é significativo do momento de amadurecimento que impõe novas reflexões sobre as articulações trabalho e educação sob diferentes ângulos de análise.

\section{CONSIDERAÇÕES FINAIS}

Para finalizar é importante considerar os limites do estudo, inicialmente por constituir-se como interpretação de um grupo intencionalmente movido por uma preocupação em problematizar os discursos sobre o cuidado; também por focalizar-se sobre um corpus de artigos que, embora tratados por meios sistemáticos de busca e análise, não representa toda a possível amplitude e complexidade desta produção. Tampouco o estudo pôde se fixar em procedimentos de uma 
revisão integrativa de literatura, uma vez que não podia se restringir a resultados de pesquisa, mas devia privilegiar proposições teóricas sobre o cuidado, muitas vezes presentes em artigos de reflexão. Apesar desses limites, a análise das produções acerca do cuidado nos permitiu identificar a relevância desta temática na área da saúde, e principalmente na Enfermagem, após a década de 80, destacando-se, neste contexto, a relação do cuidado com a dimensão do trabalho, mas também com as dimensões educativa e política. Cuidado que ultrapassa a simples ação/fazer, e que se constitui como categoria fundamental no processo de trabalho em saúde, sendo alvo de reflexões de diferentes profissionais de saúde, mesmo que neste artigo o corpus central tenha se constituído de produções de enfermeiras. ${ }^{16}$

Há que se destacar o cuidado como objeto epistemológico da enfermagem, o que justifica a significativa produção sobre esta temática por estes profissionais. Um cuidado na perspectiva da integralidade, alicerçado na práxis educativa e como princípio político, o que se verificou em diferentes reflexões. Essa relação do trabalho, educação e política, com a práxis do cuidado, se coloca em uma perspectiva transformadora.

Neste contexto de transformação, as reflexões da enfermagem sobre o cuidado identificam-se com as políticas públicas, pautadas nos princípios do Sistema Único de Saúde, de defesa da integralidade e da eqüidade, reforçando a dimensão política do trabalho "cuidar". Um cuidar que se articula à educação, visando o resgate da cidadania, em que o sujeito do cuidado e o cuidador estejam em sintonia, o que reforça o pensamento da educação como dimensão inerente ao cuidado e ao trabalho. Há, portanto, que se realizar um cuidado respaldado no conhecimento produzido, visando responder aos anseios dos profissionais, às necessidades de cuidado da população e a um cuidado que busque a integralidade no âmbito do trabalho coletivo em saúde.

\section{REFERENCIAS}

1. Germano RM. Educação e ideologia da enfermagem no Brasil. São Paulo (SP): Cortez; 1984.

2. Almeida MCP. O saber de enfermagem e sua dimensão prática. São Paulo (SP): Cortez; 1986.

3. Silva GB. Enfermagem profissional: análise crítica. São Paulo (SP): Cortez; 1986.

4. Pires D. Hegemonia médica na saúdee a enfermagem. São Paulo (SP): Cortez; 1989
5. Zago AT. O cuidado na perspectiva da Associação Brasileira de Enfermagem - Seção Santa Catarina. Texto Contexto Enferm. 1988 Mai-Ago; 7(2):179.

6. Machado FRS, Pinheiro R, Guizardi FL. As novas formas de cuidado integral nos espaços públicos de saúde. In: Pinheiro R, Matos RA, organizadores. Cuidado: as fronteiras da integralidade. Rio de Janeiro (RJ): HUCITEC/ ABRASCO; 2004. p.57-74.

7. Campos GWS. Subjetividade e administração de pessoal: considerações sobre os modos de gerenciar o trabalho de equipe de saúde. In: Merhy EE, Onocko $\mathrm{R}$, organizadores. Agir em saúde: um desafio para o público. 2a . ed. São Paulo (SP): HUCITEC; 2003.

8. Favoreto CAO. A velha e renovada clínica dirigida à produção de um cuidado integral em saúde. In: Pinheiro R, Matos RA, organizadores. Cuidado: as fronteiras da integralidade. Rio de Janeiro (RJ): HUCITEC/ABRASCO; 2004. p. 205-19.

9. Leopardi MT, Gelbcke FL, Ramos FRS. Cuidado: objeto de trabalho ou objeto epistemológico da enfermagem?. Texto Contexto Enferm. 2001 JanMar; 10(1):32-49.

10. Crossetti MGO, Buogo M, Kohlraush E. O cuidar de enfermagem como ação terapêutica na atenção a saúde individual: a especificidade das ações de natureza propedêuticas e terapêuticas de enfermagem e sua interfaze com as ações de enfermagem complementares aos atos de outros profissionais In: Anais do $50^{\circ}$. Congresso Brasileiro de Enfermagem, 199820 a 26 de setembro de 1998; Salvador, Brasil. Salvador (BA): Aben-seção Bahia; 1999. p.94-111.

11. Sa LD. E a enfermagem do século XXI? Rev Bras Enferm. 1999 Jul-Set; 52(3):375-84.

12. Mishima SM, Almeida MCP, Pinto IC, Villa TCS, Anselmi ML. Reflexões acerca da gerência do cuidado de enfermagem na rede básica de saúde. In: Anais do $50^{\circ}$. Congresso Brasileiro de Enfermagem, 20 a 26 de setembro de 1998; Salvador, Brasil. Salvador (BA): Aben-seção Bahia; 1999. p.179-180.

13. Pomatti DM, Ramos IR, Bettinelli LA. Cuidado de enfermagem: um compromisso social. Rev Medica HSVP. 1997; 9(23):27-30.

14. Neves EP. Cuidando e confortando: uma estratégia para a saúde do trabalhador. Rev Enferm UERJ. 2002 Mai-Ago; 10(2):133-7.

15. Santin S. Cuidado e/ou conforto: um paradigma para a enfermagem, desenvolvido segundo o costume dos filósofos. Texto Contexto Enferm. 1998 Abr-Jun; 7(2):111-32.

16. Ayres JRCM. O cuidado, os modos de ser (do) humano e as práticas de saúde. Saúde Soc. 2004 Set-Dez; 13(3):16-29.

17. Silva AL. Cuidado como momento de encontro e troca. In: Anais do 50 ${ }^{\circ}$. Congresso Brasileiro de Enfermagem, 20 a 26 de setembro de 1998; Salvador, Brasil. Salvador (BA): Aben-seção Bahia; 1999. p.74-9. 
18. Waldow VR. Cuidar como marco de referência para o ensino da enfermagem In: Anais do $50^{\circ}$. Congresso Brasileiro de Enfermagem, 20 a 26 de setembro de 1998; Salvador, Brasil. Salvador (BA): Aben-seção Bahia; 1999. p.197-201.

19. Wendhausen ALP. Assistência de Enfermagem: da sujeição dos corpos à autonomia do sujeito. Cogitare Enferm. 1997 Jan-Jun; 2(1):25-8.
20. Waldow VR. O cuidar humano: reflexões sobre o processo de enfermagem versus processo de cuidar. Rev Enferm. UERJ. 2001 Set-Dez; 9(3):284-93.

21. Gil TG, Gimeno LM, Gonzales RL. Antropología de los cuidados en el ámbito académico de la enfermería en España. Texto Contexto Enferm. 2006 Jan-Mar; 15(1):155-61. 